\title{
LDPC Code Design for Two-Layer Spatial Modulation
}

\author{
José Clair Menezes Júnior, Bruno Fontana da Silva and Bartolomeu F. Uchôa Filho
}

\begin{abstract}
In Spatial Modulation (SM), the information bits are split into two streams: one is mapped to a conventional complex signal modulation, and the other is used to select the active transmit antenna. Transmission SM schemes can be implemented with a single RF chain, allowing for a low-complexity transmitter with relatively large spectral efficient. In this paper, we are concerned with coded SM schemes. In the literature, the usual approach considers a single encoder that jointly encodes both bit streams. In this work, we propose a coded SM system in which each of the information streams is encoded by a possibly different low-density parity-check (LDPC) code. The two LDPC codes are designed after selecting the code rates according to the mutual information associated with the two corresponding subchannels, for a fixed, reference signal-to-noise ratio (SNR). A two-layer, iterative message-passing decoder is developed, where the two component decoders exchange soft information. Through Monte Carlo simulations, we show that the bit error rate of the proposed scheme is low when the SNR is greater or equal to the reference $\mathrm{SNR}$, demonstrating the strength of the proposed two-layer approach.
\end{abstract}

Index Terms-LDPC codes, multiple-input multiple-output (MIMO), spatial modulation (SM).

\section{INTRODUCTION}

W IRELESS communication systems using multiple transmit and receive antennas, i.e., multiple-input, multiple-output (MIMO) systems, have received wide attention in the last decades [2], [3], [4], as they are capable of achieving considerable gains in terms of diversity [3], [4] and/or capacity [5] over the systems with single transmit and receive antenna (SISO) [1].

Recently, spatial modulation (SM) [6] has emerged as an attractive MIMO technique, where the indexes of the transmit antennas are exploited as an additional dimension. Using the antennas indexes, further information can be conveyed, improving the spectral efficiency.

One of the main advantages of SM comes from the fact that only a single antenna is active at a given time, which requires a single radio frequency (RF) chain at the transmitter. Hence, the transmitter complexity is reduced. This contrasts with conventional MIMO techniques, such as space-time coding [4]

J. C. Menezes Júnior is with Federal Institute Sul-rio-grandense (IFSul), Pelotas-RS, Brazil, e-mail: josemenezes@ pelotas.ifsul.edu.br. B. F. da Silva is with Federal Institute Sul-rio-grandense (IFSul), Sapiranga-RS, Brazil, email: brunosilva@ifsul.edu.br.

B. F. Uchôa-Filho is with Department of Electrical and Electronics Engineering, Federal University of Santa Catarina, Florianópolis-SC, Brazil, email: uchoa@eel.ufsc.br.

This work was supported in part by The Coordination for the Improvement of Higher Education Personnel (CAPES) - Finance Code 001, and by The Brazilian National Council for Scientific and Technological Development (CNPq) .

Digital Object Identifier: 10.14209/jcis.2019.23 and spatial multiplexing [5], for which the required number of RF chains is equal to the number of transmit antennas [7].

In order to improve the Bit Error Rate (BER) performance in these systems, several works have considered the use of error-correcting codes for SM, generally fitting into two types of approach. In the so-called one-layer type, the most common approach, the two information streams (i.e., the ones associated with the conventional and spatial modulations) are jointly encoded. Under this approach, trellis coded modulation [8], [9], turbo codes [10], and LDPC (low-density paritycheck) codes [11], [12], [13] have been designed.

In the second approach, a two-layer design is considered where the two information streams are encoded separately by using two different component codes. In [13], a non-binary LDPC-SM system in both one- and two-layer configurations have been shown to achieve good performance, at the expenses of a high decoding complexity due to non-binary field operations. Another two-layer scheme was proposed in [14], wherein each information sequence is encoded by a block Markov superposition transmission (BMST) scheme, with iterations between the component decoders. Through simulations, the system has been shown to achieve good BER performance over uncorrelated Rayleigh fading channels. However, the SMBMST scheme in [14] has high decoding complexity, also requiring large encoding memories and decoding window sizes [15].

To circumvent the problem of high decoding complexity of the previous schemes, in this paper we propose a twolayer SM scheme with binary LDPC [16] encoders for each information stream, which allows for a simple iterative decoder structure. We observe that, conditioned on the channel's output observations, the a priori information of the bits related to the signal modulation can be used to obtain the a posteriori probabilities of the bits related to the spatial modulation, and vice-versa. Therefore, iterations between the two component decoders are possible and, in fact, improve the reliability of both bit streams. We exploit this fact in the proposed iterative decoder.

In a two-layer coded scheme, the code rates of the two codes must be selected according to some criterion. In this work, we numerically evaluate the mutual information between input signals and the received signal as function of the signalto-noise ratio (SNR), and use this quantity to select the two component code rates. With the proper code rates, we design the binary irregular component LDPC codes [17], where the degree distributions are designed to improve the BER performance under the sum-product decoding algorithm (SPA). Simulations results in uncorrelated Rayleigh channels demonstrate a significant improvement in BER performance 
with respect to the one-layer scheme.

The contributions of this work are:

- Design and implementation of a two-layer binary LDPC coding scheme for SM, where the two information bit streams (associated with spatial and conventional modulations) are separately encoded.

- The evaluation of the mutual information of the two transmitted bit streams and the received signal as a way to select the component code rates. The suitability of this selection is verified through Monte Carlo simulation. It is shown that, under proper rates selection, the proposed binary coded SM scheme performs well with relatively low decoding complexity.

- Proposition of a strategy for message exchange between the two component SPAs. Through simulations, it is demonstrated the superior performance of the proposed method in comparison with a two-layer scheme without this strategy.

The remainder of this paper is organized as follows. In Section II, the classification of SM systems and proposed SM-coded system model are described. Aspects about LDPC encoding processes and the assignment of code rates are demonstrated in Section III. In Section IV, detection/decoding processes are presented. In Section V, the simulation results are demonstrated. Finally, Section VI concludes the paper.

Notation: The operation $(.)^{T}$ denotes the transpose of a vector or matrix, $\|\cdot\|$ denotes the Euclidean norm. The scalars, column vectors, matrices and sets are denoted by lower-case, bold-face lower-case, boldface upper-case, and calligraphic letters $(d, \mathbf{d}, \mathbf{D}$ and $\mathcal{D})$, respectively.

\section{Coded Spatial Modulation}

\section{A. Classification}

In general, coded spatial modulation has been divided into one-layer and two-layer approaches, which can be described as:

- One-layer: the information vector $\mathbf{u}$ is encoded into a sequence c. Then, c is used for SM transmission.

- Two-layer: the information vector $\mathbf{u}$ is first divided into two sub-vectors: $\mathbf{u}_{\mathrm{a}}$ and $\mathbf{u}_{\mathrm{s}}$. The vector $\mathbf{u}_{\mathrm{a}}$ is encoded into the vector $\mathbf{c}_{\mathrm{a}}$ which is considered for the spatial component of the SM signal. The bit vector $\mathbf{u}_{\mathrm{s}}$ is independently encoded into the vector $\mathbf{c}_{\mathrm{s}}$. This coded vector then passes through an $M$-ary signal modulator, producing the vector $\mathbf{x}$. The two vectors $\mathbf{c}_{\mathrm{a}}$ and $\mathbf{x}$ are used for SM transmission.

Based on the current literature, we herein refine this classification by subdividing the two-layer approach described above into the two-layer partial and two-layer total approaches. The difference between them is that, in the former, only the bit stream intended for the spatial component (i.e., $\mathbf{u}_{\mathrm{a}}$ ) is encoded, while $\mathbf{u}_{\mathrm{s}}$ is left uncoded. In Table I, related works on coded SM are listed according to the approach they follow and the corresponding configurations are illustrated in Fig. 1.

In [11], LDPC codes are optimized in a SM-MIMO system under the one-layer approach by employing the extrinsic
TABLE I

RELATED WORKS ON CODED SM

\begin{tabular}{|c|c|c|}
\hline One-layer & Two-layer partial & Two-layer total \\
\hline E. Başar et al. [9], & R. Mesleh et al. [8], & L. Wang et al. [14]. \\
H. Liu et al. [11], & C. Vladeanu [10], & D. Feng et al.[13]. \\
D. Feng et al. [13], & L. Wang e X. Ma [18], & \\
X. Q. Jiang et al. [12]. & & \\
\hline
\end{tabular}

information transfer (EXIT) chart technique. In [13], the nonbinary LDPC coding with Gallager mapping was used also under the one-layer approach to eliminate the restriction of the number of active transmit antennas being a power of 2 . The two-layer total approach was also employed in order to reduce the finite field size and consequently reduce the complexity of transmission and decoding. A good trade-off between complexity and performance was demonstrated by simulation. However, BER degradation of the two-layer approach has been observed with respect to the one-layer approach.

In [14], a SM-MIMO system is proposed with BMST encoding where two transmit antennas are enabled and higher order modulation are utilized in the two-layer total approach.
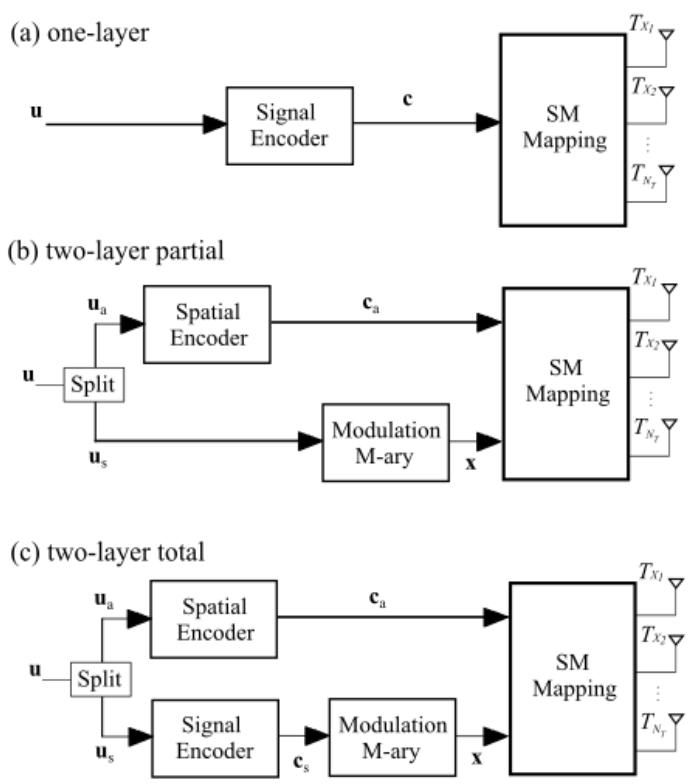

Fig. 1. Configurations in different approaches for coded SM systems.

\section{B. System Model}

Let us consider the SM-MIMO system with $N_{\mathrm{T}}$ transmit and $N_{\mathrm{R}}$ receive antennas, which is illustrated in Fig. 2. An information sequence $\mathbf{u}$ with $k$ bits is divided into two sub-blocks. The first sub-block $\mathbf{u}_{\mathrm{a}}$ passes through an LDPC encoder with code rate $R_{\mathrm{a}}$ that generates the codeword $\mathbf{c}_{\mathrm{a}}$. The second sub-block $\mathbf{u}_{\mathrm{s}}$ also passes through an LDPC encoder, with code rate $R_{\mathrm{s}}$, which generates the vector $\mathbf{c}_{\mathrm{s}}$.

Then, the binary vector $\mathbf{c}_{\mathrm{s}}$ is split into blocks of $b$ bits. According to the bits values, each block is mapped into a 


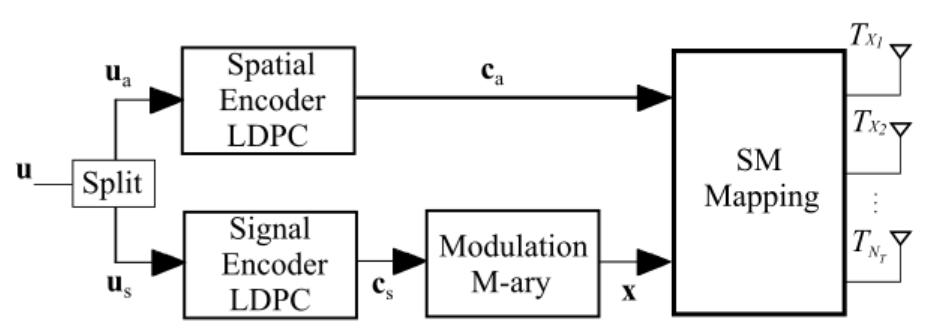

Fig. 2. Diagram of two-layer coded SM system .

signal, $s_{q}$, from a conventional $M$-ary signal modulation, phase-shift keying (PSK) or quadrature amplitude modulation (QAM), where $M=2^{b}$. The proposed system is referred to as the two-layer LDPC-SM system (2-LDPC-SM) and its spectral efficiency (in bits/s/Hz) is given by

$$
\eta=R_{\mathrm{s}} \times \log _{2} M+R_{\mathrm{a}} \times \log _{2} N_{\mathrm{T}}
$$

where $\log _{2} N_{T}$ is the number of bits per symbol period associated with the spatial component of SM, since the spatial information is related to the activation, at each symbol period, of a different transmit antenna among the $N_{T}$ transmit antennas available, while all the other antennas remain silent.

Consider the MIMO channel $\mathbf{H}_{\mathrm{ch}} \in C^{N_{\mathrm{R}} \times N_{\mathrm{T}}}$, where $\mathbf{H}_{\mathrm{ch}}=$ $\left[\mathbf{h}_{1}, \mathbf{h}_{2}, \ldots, \mathbf{h}_{N_{\mathrm{T}}}\right]$ and $\mathbf{h}_{i}=\left[h_{1 i}, h_{2 i}, \ldots, h_{N_{\mathrm{R}} i}\right]^{T}$. In the matrix $\mathbf{H}_{\text {ch }}$, each vector is modeled as complex Gaussian, independent, circularly symmetric, with zero mean and unit variance. We assume that the channel is frequency-flat with Rayleigh fading, remaining unchanged in the transmission of a joint symbol (signal and spatial components).

The SM transmit signal vector $\mathbf{x} \in C^{N_{\mathrm{T}} \times 1}$ is expressed by $\mathbf{x}=\left[0, \ldots, s_{q}, \ldots, 0\right]^{T}$, i.e., the $q$-th symbol from the signal constellation is transmitted from the $j$-th transmit antenna. So, the received signal is given by:

$$
\mathbf{y}=\mathbf{H}_{\mathrm{ch}} \mathbf{x}+\mathbf{n}=\mathbf{h}_{j} s_{q}+\mathbf{n},
$$

where $\mathbf{y} \in C^{N_{\mathrm{R}} \times 1}$ and the vector $\mathbf{n}$ is the additive white Gaussian noise, whose entries are independent and identically distributed (i.i.d.), with zero mean and variance $\left(\frac{N_{0}}{2}\right)$ per complex dimension.

At the receiver, we assume full knowledge of the path gains. The spatial and signal detector blocks refer, respectively, to the symbols of the spatial and signal vectors, and employ the MAP (maximum a posteriori) algorithm, which operates bit after bit. These blocks generate a posteriori probabilities, acting in the form of soft inputs to the SPA decoder, where these probabilities are processed among the variable and parity nodes, in the linear domain.

After processing in each SPA decoder, an iterative message passing algorithm is performed to exchange spatial and signal bits messages between the decoders. Finally, after obtaining the pseudo-probabilities of the information bits, a decision generates $\hat{\mathbf{u}}_{\mathrm{a}}$ and $\hat{\mathbf{u}}_{\mathrm{s}}$, which are grouped in the vector $\hat{\mathbf{u}}$.

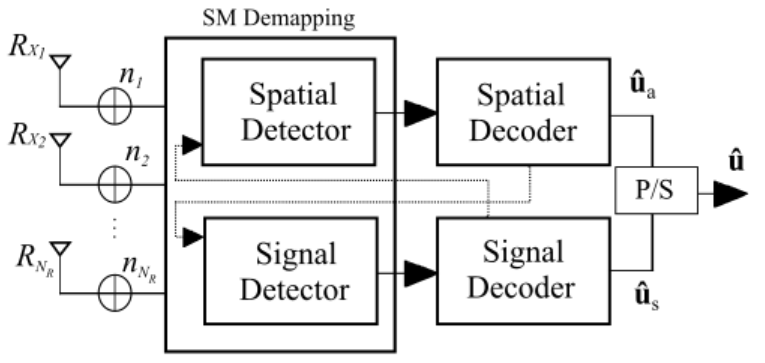

\section{LDPC-CODED SM TRANSMITTER}

\section{A. Encoding}

In general, systematic LDPC codes based on a generator matrix $\mathbf{G}$ imply great encoding complexity, due to the nonsparsity of G. Several works have been developed to find ways to reduce complexity and increase coding speed [19], [20], [21].

In the present work, the coding used in [22] is employed, in which restrictions are considered in the construction of the matrix $\mathbf{H}$, being divided into two sub-matrices $\mathbf{H}_{1}$ and $\mathbf{H}_{2}$. The sub-matrix $\mathbf{H}_{2}$ is constructed based on the quasicyclic (QC-LDPC) matrices [23]. With the knowledge of $\mathbf{H}_{2}$, constraints are imposed on the construction of $\mathbf{H}_{1}$ are imposed so that the parity matrix $\mathbf{H}$ does not contain low-length cycles and has the desired degree distribution.

It is important to point out that this coding technique is applied in the formation of the codewords $\mathbf{c}_{a}$ (spatial branch) and $\mathbf{c}_{s}$ (signal branch), and presents as characteristics: flexibility in the choice of LDPC rates, the low complexity involved in the processes since the matrix $\mathbf{H}$ is sparse, and the matrix $\mathbf{H}_{2}$ has no cycles of length 4 and has a few cycles of length 6 .

\section{B. Rate Selection for the Proposed LDPC-Coded SM System}

The Rayleigh fading channel capacity using spatial modulation was derived in [24], where a continuous Gaussian distribution was used for the source signals. It is known that Gaussian inputs are optimal for this channel. However, they are not feasible in practical realizations. For a finite input alphabet, such as $M$-QAM and $M$-PSK, the mutual information must be evaluated and it can be considereably smaller. The mutual information for SM systems is analyzed for one receive antenna in [25] and for multiple receive antennas in one-layer configuration in [26].

As discussed earlier, in spatial modulation the $j$-th transmit antenna is selected to send the symbol $s_{q}$ and the received signal is given by (2). Note that, due to the noise vector $\mathbf{n}$, although the inputs of the channel are discrete, the output $y$ is a continuous random variable.

Although in SM schemes the effective signals are transmitted only by signal modulation, it is noticeable the existence of two distinct channels, i.e., a channel used for transmission of signal modulation and a channel used by spatial modulation. Each one of the two channels has its own mutual information. 
Thus it is interesting to evaluate them, in terms of spectral efficiency, as a function of the SNR. With this metric, channel coding can be used aiming to increase reliability.

The mutual information between the inputs and output variables are evaluated through Monte Carlo simulations. The mutual information $I(S, A ; Y)$ of the two random variables ( $S$ e $A$ ), which respectively correspond to the inputs $s$ (signal) and $a$ (antenna index), with the output variable $(Y)$, which describes $\mathbf{y}$, can be described by the expressions of the chain rule of mutual information as [27]:

$$
\begin{aligned}
I(S, A ; Y) & =I(A ; Y \mid S)+I(S ; Y) \\
& =I(S ; Y \mid A)+I(A ; Y) .
\end{aligned}
$$

Once this mutual information is obtained, the selection of LDPC code rates in the two-layer system has to consider the following upper bound:

$$
\eta=b_{\mathrm{s}} R_{\mathrm{s}}+b_{\mathrm{a}} R_{\mathrm{a}} \leq I(S, A ; Y),
$$

where $b_{\mathrm{a}}$ and $b_{\mathrm{s}}$ are, respectively, the number of bits conveyed by a symbol of the spatial and signal vectors. So, we have:

$$
R_{\mathrm{s}} \leq \frac{I(S ; Y)}{b_{\mathrm{s}}} \quad \text { and } \quad R_{\mathrm{a}} \leq \frac{I(A ; Y \mid S)}{b_{\mathrm{a}}}
$$

or

$$
R_{\mathrm{s}} \leq \frac{I(S ; Y \mid A)}{b_{\mathrm{s}}} \quad \text { and } \quad R_{\mathrm{a}} \leq \frac{I(A ; Y)}{b_{\mathrm{a}}}
$$

The mutual information between the two inputs $(S$ and $A$ ) with the output $(Y)$ is given by:

$I(S, A ; Y)=\sum_{j \in \mathcal{K}} \sum_{s_{q} \in \mathcal{X}} \int_{\mathbf{y}} p\left(\mathbf{y}, \mathbf{h}_{j}, s_{q}\right) \log _{2}\left(\frac{p\left(\mathbf{y} \mid \mathbf{h}_{j}, s_{q}\right)}{p(\mathbf{y})}\right) d y$

where $\mathcal{K}$ is the spatial constellation alphabet, i.e., the set of transmit antenna indexes, and the alphabet $\mathcal{X}$ covers the set of symbols of the signal constellation. So, we have:

$$
\begin{aligned}
& I(S, A ; Y)= \\
& \log _{2} N_{\mathrm{T}} M-\frac{1}{N_{\mathrm{T}} M} \sum_{j \in \mathcal{K}} \sum_{s_{q} \in \mathcal{X}} \int_{y}\left(\frac{1}{\pi \sigma^{2}}\right)^{N_{\mathrm{R}}} \mathrm{e}^{\left(-\frac{\left|\mathbf{y}-\mathbf{h}_{j} s_{q}\right|^{2}}{\sigma^{2}}\right)} \\
& \log _{2}\left(\sum_{j_{2} \in \mathcal{K}} \sum_{s_{q_{2}} \in \mathcal{X}} \mathrm{e}^{\left(-\frac{\left|\mathbf{y}-\mathbf{h}_{j} s_{q}\right|^{2}-\left|\mathbf{y}-\mathbf{h}_{j_{2}} s_{2}\right|^{2}}{\sigma^{2}}\right)}\right) d y .
\end{aligned}
$$

The first term in the mutual information in (3) can be expressed as:

$$
\begin{aligned}
& I(A ; Y \mid S)=\log _{2} N_{\mathrm{T}}-\frac{1}{N_{\mathrm{T}} M} \sum_{j \in \mathcal{K}} \sum_{s_{q} \in \mathcal{X}} \int_{\mathbf{y}}\left(\frac{1}{\pi \sigma^{2}}\right)^{N_{\mathrm{R}}} \\
& \mathrm{e}^{\left(-\frac{\left|\mathbf{y}-\mathbf{h}_{j} s_{q}\right|^{2}}{\sigma^{2}}\right)} \log _{2}\left(\sum_{j_{2} \in \mathcal{K}} \mathrm{e}^{-\frac{\left|\mathbf{y}-\mathbf{h}_{j} s_{q}\right|^{2}-\left|\mathbf{y}-\mathbf{h}_{j_{2}} s_{q}\right|^{2}}{\sigma^{2}}}\right) d y .
\end{aligned}
$$

Performing some algebraic manipulations, the mutual information between the input $S$ and the output $Y$ is given by:

$$
\begin{aligned}
& I(S ; Y)= \\
& \log _{2} M-\frac{1}{N_{\mathrm{T}} M} \sum_{j \in \mathcal{K}} \sum_{s_{q} \in \mathcal{X}} \int_{\mathbf{y}}\left(\frac{1}{\pi \sigma^{2}}\right)^{N_{\mathrm{R}}} \mathrm{e}^{\left(-\frac{\left|\mathbf{y}-\mathbf{h}_{j} s_{q}\right|^{2}}{\sigma^{2}}\right)} \\
& \log _{2}\left(\frac{\sum_{j_{2} \in \mathcal{K}} \sum_{s_{q_{2}} \in \mathcal{X}} \mathrm{e}^{\left(-\frac{\left|\mathbf{y}-\mathbf{h}_{j} s_{q}\right|^{2}-\mid \mathbf{y}-\mathbf{h}_{\left.j_{2} s_{2}\right|^{2}}}{\sigma^{2}}\right)}}{\sum_{j_{2} \in \mathcal{K}}}\right) d y .
\end{aligned}
$$

The expressions for calculation of the mutual information $I(S ; Y \mid A)$ and $I(A ; Y)$ are computed as, respectively:

$$
\begin{aligned}
& I(S ; Y \mid A)= \\
& \log _{2} M-\frac{1}{N_{\mathrm{T}} M} \sum_{j \in \mathcal{K}} \sum_{s_{q} \in \mathcal{X}} \int_{\mathbf{y}}\left(\frac{1}{\pi \sigma^{2}}\right)^{N_{\mathrm{R}}} \mathrm{e}^{\left(-\frac{\left|\mathbf{y}-\mathbf{h}_{j} s_{q}\right|^{2}}{\sigma^{2}}\right)} \\
& \log _{2}\left(\sum_{s_{q 2} \in \mathcal{X}} \mathrm{e}^{\left(-\frac{\left|\mathbf{y}-\mathbf{h}_{j} s_{q}\right|^{2}-\left|\mathbf{y}-\mathbf{h}_{j} s_{q_{2}}\right|^{2}}{\sigma^{2}}\right)}\right) d y,
\end{aligned}
$$

and,

$$
\begin{aligned}
& I(A ; Y)= \\
& \log _{2} N_{\mathrm{T}}-\frac{1}{N_{\mathrm{T}} M} \sum_{j \in \mathcal{K}} \sum_{s_{q} \in \mathcal{X}} \int_{\mathbf{y}}\left(\frac{1}{\pi \sigma^{2}}\right)^{N_{\mathrm{R}}} \mathrm{e}^{\left(-\frac{\left|\mathbf{y}-\mathbf{h}_{j} s_{q}\right|^{2}}{\sigma^{2}}\right)} \\
& \log _{2}\left(\frac{\sum_{j_{2} \in \mathcal{K}} \sum_{s_{q 2} \in \mathcal{X}} \mathrm{e}^{\left(-\frac{\left|\mathbf{y}-\mathbf{h}_{j} s_{q}\right|^{2}-\left|\mathbf{y}-\mathbf{h}_{j_{2}} s_{q 2}\right|^{2}}{\sigma^{2}}\right)}}{\sum_{s_{q 2} \in \mathcal{X}} \mathrm{e}^{\left(-\frac{\left|\mathbf{y}-\mathbf{h}_{j} s q\right|^{2}-\left|\mathbf{y}-\mathbf{h}_{j} s_{q 2}\right|^{2}}{\sigma^{2}}\right)}}\right) d y .
\end{aligned}
$$

According to (9), it can be verified that, with the increase of SNR, the term $\log _{2} N_{\mathrm{T}} M$ is predominant.

Note that the final result of (3) and (4) are identical, but, with the increase of SNR, the mutual information values in each of the component terms of the equations are distinct. In this way, it can be defined which path to select depending of the desired constraints.

\section{LPDC-Coded SM RECEIVER: Detection AND DECODING}

For simplicity, it is assumed that the bits of the signal constellation and the spatial constellation are independent and generated with equal probability. The a posteriori probability that the $v$-th bit of the antenna index vector is equal to $w$ [28], where $w \in[0,1]$, is

$$
p\left(\mathbf{u}_{\mathrm{a}}^{v}=w \mid \mathbf{y}\right)=\frac{\sum_{j \in \mathcal{K}_{w}^{v}} \sum_{s_{q} \in \mathcal{X}} p\left(\mathbf{y}, \mathbf{h}_{j}, s_{q}\right)}{p(\mathbf{y})},
$$

where $\mathcal{K}_{w}^{v}$ refers to the antennas indexes for which the $v$-th bit is equal to $w$. 
For the detection of symbols of the signal vector, the calculations are performed in a manner analogous to (14). Thus, we compute the a posteriori probability that the $v$-th bit of $\mathbf{u}_{\mathrm{s}}$ is equal to $w$ as:

$$
p\left(\mathbf{u}_{\mathrm{s}}^{v}=w \mid \mathbf{y}\right)=\frac{\sum_{s_{q} \in \mathcal{X}_{w}^{v}} \sum_{j \in \mathcal{K}} p\left(\mathbf{y}, \mathbf{h}_{j}, s_{q}\right)}{p(\mathbf{y})},
$$

where $\mathcal{X}_{w}^{v}$ refers the data symbols for which the $v$-th bit is equal to $w$.

Thereafter, the results of the calculations of the above expressions are used as input to the SPA decoders of each corresponding information vector. The estimated information vector $\hat{\mathbf{u}}$ is obtained by completing the iterations in the SPA decoders or obtaining a valid codeword. The iterative decoding with information exchange follows the steps described in Algorithm 1.

Then, we considered the influence of the a priori probability of the bits of the signal modulation to find the a posteriori probability of the bits related to the indexes of the antennas, and vice-versa. This is relevant since, in general, for the evaluation of the a posteriori probability of the information bit in question, the a priori probability itself is considered [29].

In this way, we explore in the present work the use of the extrinsic information generated at the output of the SPA decoder for the LDPC code used in the signal modulation to provide a priori probabilities for the input of the detector of the LDPC code used for spatial modulation, and vice-versa. That is, there is an update of the a priori probabilities of the information bits (either spatial or signal), via message exchange between the SPA decoders.

It should be noted, from (14) and (15), that the detection complexities of the spatial and the conventional modulation components are essentially the same. The same applies to the decoding complexity. Therefore, we can say that the complexity of each iteration of the SPA detector/decoder for one- or two-layer as well as for the conventional LDPC code is essentially the same.

We also note that there is no difference in complexity between the two-layer scheme with no iteration $\left(i_{D}=0\right)$ between the SPA decoders and the one with one iteration $\left(i_{D}=1\right)$, as the only difference between these cases is that in the latter one SPA component decoder feeds the other with some soft information, whereas in the former the two SPA component decoders operate independently. On the other hand, the overall decoding complexity of the two-layer scheme increases proportionally to $i_{D}$ for $i_{D} \geq 1$. Nevertheless, as we will see from the simulation results, this complexity increase is justifiable.

\section{Simulation Results}

In this section, the BER of the proposed coded scheme as a function of the SNR is evaluated through Monte Carlo simulations. Uncorrelated Rayleigh fading is considered for the channel model in (2). The fading coefficients are constant during the transmission of each joint symbol and varies of from one symbol to another. We consider two scenarios under which the proposed two-layer total scheme with iteration $\overline{\text { Algorithm } 1 \text { Iteration between detection and decoding pro- }}$ cesses in two-layer system

I) Initialization: Define the maximum number of iterations between decoders $\left(i_{\mathrm{D}_{\max }}\right)$ and the uniform distribution of the a priori probabilities of the signal constellation $P\left(s_{q}\right)$ and spatial constellation $P\left(\mathbf{h}_{j}\right)$.

II) Detection/decoding: For $i_{\mathrm{D}}=1,2, \ldots, i_{\mathrm{D}_{\max }}$, - Step 0: Signal detector

Calculate the a posteriori probabilities associated with the $v$-th bit of the vector $\mathbf{u}_{\mathrm{s}}$,

$$
p\left(\mathbf{u}_{\mathrm{s}}^{v}=w \mid \mathbf{y}\right)=\sum_{s_{q} \in \mathcal{X}_{w}^{v}} \sum_{j \in \mathcal{K}} \frac{p\left(\mathbf{y} \mid \mathbf{h}_{j}, s_{q}\right) P\left(s_{q}\right) P\left(\mathbf{h}_{j}\right)}{P(\mathbf{y})},
$$

\section{- Step 1: Signal decoder}

With the probabilities $p\left(\mathbf{u}_{\mathrm{s}}^{v}=w \mid \mathbf{y}\right)$ coming from the signal detector, iterations are performed in the SPA decoder and the update of the extrinsic messages $p^{\mathrm{e}}\left(\mathbf{u}_{\mathrm{s}}^{v}=w\right)$ is considered as updated entries in the spatial detector in the form $P\left(s_{q}\right)$.

\section{- Step 2: Spatial detector}

Calculate the a posteriori probabilities associated with the $v$-th bit of the vector $\mathbf{u}_{\mathrm{a}}$,

$$
p\left(\mathbf{u}_{\mathrm{a}}^{v}=w \mid \mathbf{y}\right)=\sum_{j \in \mathcal{K}_{w}^{v}} \sum_{s_{q} \in \mathcal{X}} \frac{p\left(\mathbf{y} \mid \mathbf{h}_{j}, s_{q}\right) P\left(s_{q}\right) P\left(\mathbf{h}_{j}\right)}{P(\mathbf{y})} .
$$

\section{- Step 3: Spatial decoder}

With the probabilities $p\left(\mathbf{u}_{\mathrm{a}}^{v}=w \mid \mathbf{y}\right)$ coming from the spatial detector, iterations are performed in the spatial decoder SPA and the update of the extrinsic messages $p^{\mathrm{e}}\left(\mathbf{u}_{\mathrm{a}}^{v}=w\right)$ is considered as updated inputs to the signal detector in the form $P\left(\mathbf{h}_{j}\right)$.

\section{III) Decisor}

If $i_{\mathrm{D}_{\max }}$ is reached, the estimated vector $\hat{\mathbf{u}}_{\mathrm{s}}$ is delivered at the output of the signal decoder and the output of the spatial decoder generates estimated vector $\hat{\mathbf{u}}_{\mathrm{a}}$.

between SPA decoders is compared to other one- and twolayer schemes under the same spectral efficiency.

All the BER results shown in the figures are based on the weighted average between the BERs of the two LDPC codes (when aplicable), taking into account the $M$-ary signal modulation. More specifically,

$$
\mathrm{BER}_{\text {overall }}=\frac{\left(\mathrm{BER}_{\mathrm{s}} \times R_{\mathrm{s}}+\mathrm{BER}_{\mathrm{a}} \times R_{\mathrm{a}}\right)}{\eta},
$$

where $\mathrm{BER}_{\mathrm{s}}$ and $\mathrm{BER}_{\mathrm{a}}$ are the $\mathrm{BER}$ rates of the signal and spatial vectors, respectively.

\section{A. Scenario 1}

In this scenario, we have $N_{\mathrm{T}}=2, N_{\mathrm{R}}=1$, and BPSK modulation. In Figure 3, we show the mutual information results for this scenario. We choose as operation point the SNR value of $14.8 \mathrm{~dB}$ (vertical dotted line in the figure). For this 


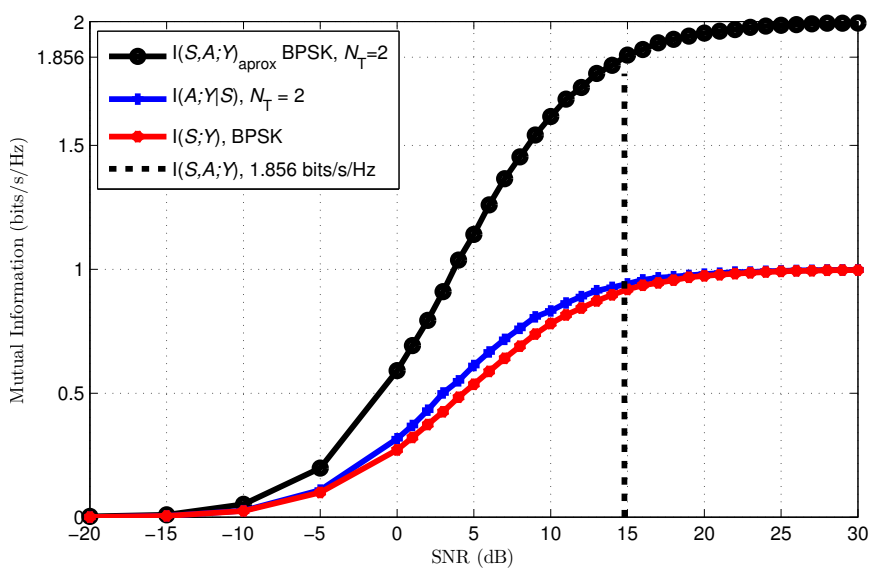

Fig. 3. Mutual information $\left(N_{\mathrm{T}}=2, N_{\mathrm{R}}=1\right.$, BPSK $)$.

SNR, the overall mutual information (i.e., maximum spectral efficiency) is $I(A, S ; Y \mid H)=1.856 \mathrm{bits} / \mathrm{s} / \mathrm{Hz}$.

We are interested in obtaining a good tradeoff between spectral efficiency and energy per bit, considering an admissible BER for wireless applications being in the range of $1 \times 10^{-6}$ and $1 \times 10^{-7}$ [30]. As this mutual information is a theoretical limit, we apply a slight rate reduction of $3.56 \%$, from 1.856 to $1.79 \mathrm{bits} / \mathrm{s} / \mathrm{Hz}$. Correspondingly, using (10) and (11), in the proposed two-layer scheme the individual code rate values $R_{\mathrm{S}}$ and $R_{\mathrm{a}}$ are proportionally reduced from 0.9165 to 0.884 bits/s/Hz and from 0.9395 to 0.906 bits $/ \mathrm{s} / \mathrm{Hz}$, respectively. In this scenario, for all schemes, we set the block length to 22400 bits and the SPA decoder performs 18 iterations. The schemes, their characteristics, and parameters are described as follows:

- 2-LDPC-SM, $i_{\mathrm{D}}=\ell$, where $\ell \in(0,1,2,3)$ : The proposed two-layer total structure with LDPC coding and $\ell$ iterations between SPA decoders, and with code rates $R_{\mathrm{s}}=0.884$ and $R_{\mathrm{a}}=0.906$. This yields a spectral efficiency of $1.79 \mathrm{bits} / \mathrm{s} / \mathrm{Hz}$.

- 1-LDPC-SM: It refers to the one-layer structure with LDPC coding on all information vector and code rate $R=0.895$. It should be remarked that half of the coded bits are used for antenna selection. Therefore, in this scheme, the spectral efficiency is $2 R=1.79 \mathrm{bits} / \mathrm{s} / \mathrm{Hz}$.

- Two-layer partial (T-L partial): It refers to the two-layer partial structure with LDPC coding only in the spatial vector with code rate $R_{\mathrm{a}}=0.79$ and uncoded for signal vector $\left(R_{s}=1\right)$. This yields a spectral efficiency of 1.79 $\mathrm{bits} / \mathrm{s} / \mathrm{Hz}$. A hard decision for signal vector is considered. Assuming zero error in the decoding of the spatial vector, we have a lower bound for the system's BER as [31]:

$$
\mathrm{BER}_{\text {low }}=\frac{1}{2 \eta}\left(1-\sqrt{\frac{\overline{\gamma_{b}}}{1+\overline{\gamma_{b}}}}\right),
$$

where $\overline{\gamma_{b}}$ is the average signal-to-noise ratio.

In Fig. 4, the BER of the proposed scheme for different numbers of iterations between SPA decoders is shown. We notice the performance improvement as the number of iterations between the two layers increases. For $i_{\mathrm{D}}>3$, the performance is kept practically unchanged. The BER curves show a good

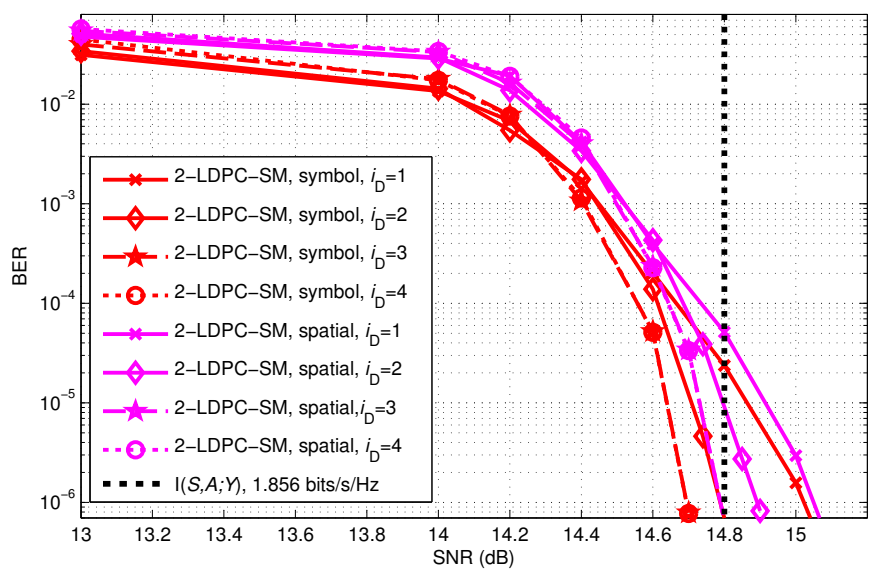

Fig. 4. Performance curves of the proposed coded SM system with various $i_{\mathrm{D}}$ values.

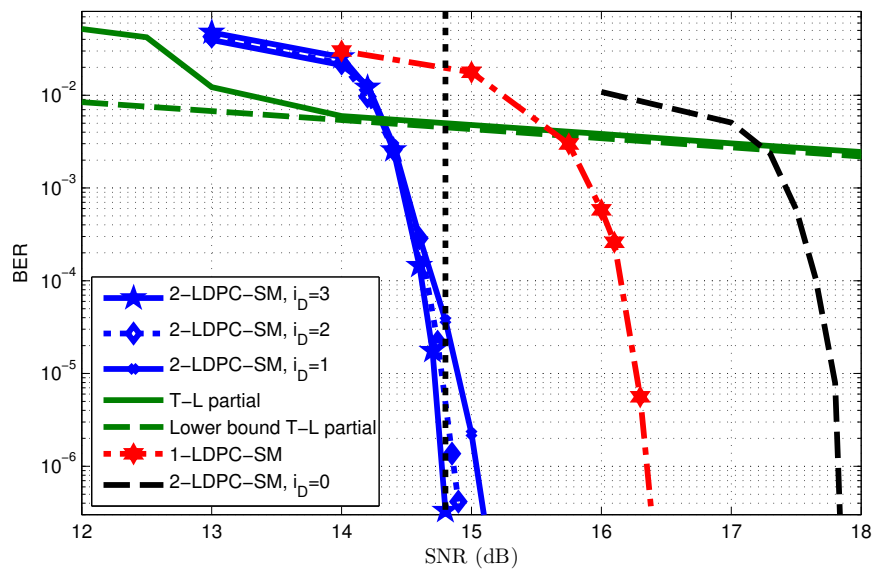

Fig. 5. Performance curves of the three schemes under Scenario 1.

performance around the operation point, especially for the 2LDPC-SM system with $i_{\mathrm{D}}=3$. Thus, the choice of slightly smaller rates was important in this case.

In Fig. 5, the three schemes (proposed and from the literature) under this scenario are compared. By comparing the curves of 2-LDPC-SM $i_{\mathrm{D}} \geq 1$ with the other curves, it is clear that the proposed system with iteration between SPA decoders outperforms all other schemes. More than that, it is the only scheme presenting a good performance at the chosen SNR. Specifically, the proposed scheme 2-LDPC-SM $i_{\mathrm{D}}=3$ achieves a BER $=4 \times 10^{-7}$ with an SNR excess of only $0.005 \mathrm{~dB}$ with regard to the reference SNR. In Table II, the SNR excess for achieving this same BER is shown for all schemes.

\section{B. Scenario 2:}

In this scenario, we have $N_{\mathrm{T}}=4, N_{\mathrm{R}}=4$, and QPSK modulation. In Figure 6, we show the mutual information results for this scenario. We choose as operation point the SNR value of $-2.8 \mathrm{~dB}$ (vertical dotted line in the figure). For this SNR, the overall mutual information is $I(A, S ; Y \mid H)=2$ bits/s/Hz, with $I(A ; Y) \approx 0.54 \mathrm{bits} / \mathrm{s} / \mathrm{Hz}$ and $I(S ; Y \mid A) \approx$ 
TABLE II

SNR EXCESS FOR ACHIEVING A BER $=4 \times 10^{-7}$ IN SCENARIO 1

\begin{tabular}{|c|c|}
\hline SM-MISO Configurations & Excess (in dB) \\
\hline 2-LDPC-SM $i_{\mathrm{D}}=3$ & 0.005 \\
\hline 2-LDPC-SM $i_{\mathrm{D}}=2$ & 0.1 \\
\hline 2-LDPC-SM $i_{\mathrm{D}}=1$ & 0.28 \\
\hline 1-LDPC-SM & 1,58 \\
\hline 2-LDPC-SM $i_{\mathrm{D}}=0$ & 3.03 \\
\hline
\end{tabular}

$1.46 \mathrm{bits} / \mathrm{s} / \mathrm{Hz}$. Thus, since $b_{s}=2$ (QPSK modulation) and $b_{a}=2$ (four transmit antennas), from (5) the corresponding code rates are, respectively, $R_{\mathrm{s}}=0.73$ and $R_{\mathrm{a}}=0.27$.

In this scenario, for all schemes, we set the block length to 2300 bits and the SPA decoder performs 50 iterations. The schemes, their characteristics, and their parameters are described as follows:

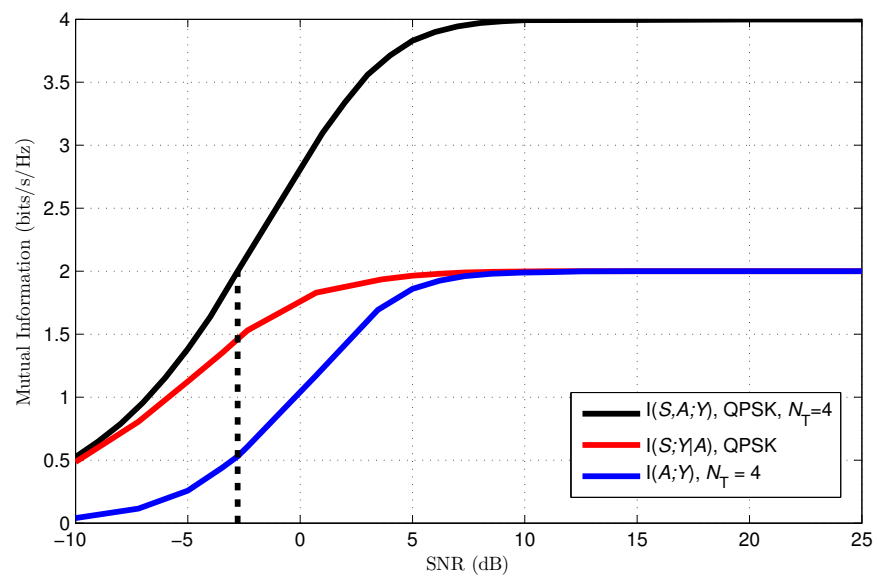

Fig. 6. Mutual information $\left(N_{\mathrm{T}}=4, N_{\mathrm{R}}=4\right.$, QPSK).

- 2-LDPC-SM, $i_{\mathrm{D}}=3$ : The proposed two-layer total structure with LDPC coding and 3 iterations between SPA decoders, and with code rates $R_{\mathrm{s}}=0.73$ and $R_{\mathrm{a}}=0.27$. This yields a spectral efficiency of $2 \mathrm{bits} / \mathrm{s} / \mathrm{Hz}$.

- 2-LDPC-SM, $\mathcal{C}(2304,1152)$ : The two-layer total structure with LDPC coding [32] and 3 iterations between SPA decoders, and with code rates $R_{\mathrm{s}}=R_{\mathrm{a}}=0.5$, yielding a spectral efficiency of $2 \mathrm{bits} / \mathrm{s} / \mathrm{Hz}$. Note that, although the overall rate respects the overall mutual information in Figure 6, the component code rate $R_{\mathrm{a}}$ does not. So, this rate selection does not follow our design rules.

- 1-LDPC-SM, $\mathcal{C}(2304,1152)$ : It refers to the one-layer structure with LDPC coding [32] on all information vector and code rate $R=0.5$. It should be remarked that half of the coded bits are used for antenna selection. Therefore, in this scheme, the spectral efficiency is also $2 \mathrm{bits} / \mathrm{s} / \mathrm{Hz}$.

In Fig. 7, the three schemes (proposed and from the literature) under this scenario are compared. Three iterations $\left(i_{D}=3\right)$ between the SPA decoders are adopted in the two two-layer schemes. The proposed 2-LDPC-SM $i_{\mathrm{D}}=3$ outperforms all other schemes. In fact, it is the only scheme that presents a reasonable performance at the reference SNR
$=-2.8 \mathrm{~dB}$. The concurrent schemes simply do not work at that low SNR. For a BER $=1 \times 10^{-6}, 2$-LDPC-SM $i_{\mathrm{D}}=3$ requires an SNR excess of $0.425 \mathrm{~dB}$. The SNR excesses for 2-LDPC-SM, $\mathcal{C}(2304,1152)$ and 1-LDPC-SM, $\mathcal{C}(2304,1152)$ are $1.04 \mathrm{~dB}$ and $3.03 \mathrm{~dB}$, respectively.

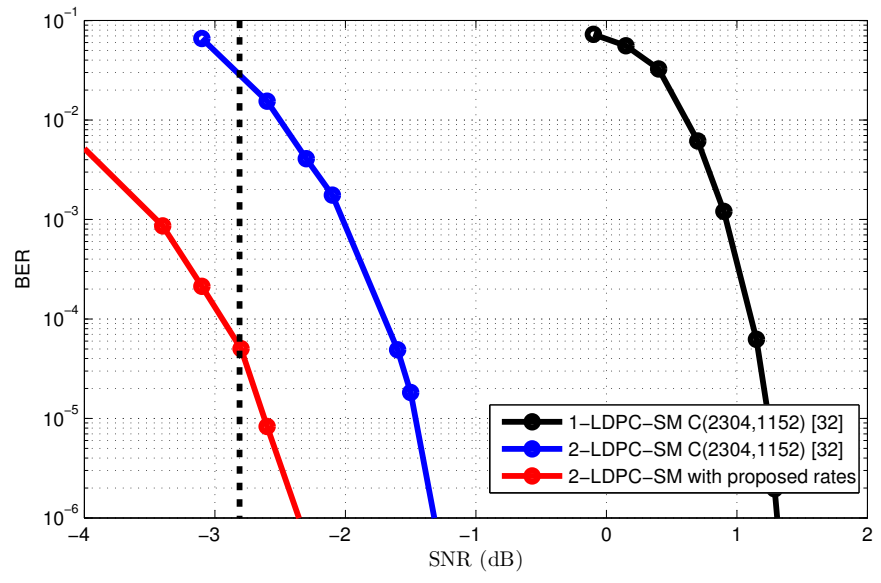

Fig. 7. Performance curves of the three schemes under Scenario 2. For the two 2-LDPC-SM schemes, $i_{D}=3$ is adopted.

\section{CONCLUSION}

In this paper, a two-layer approach for designing LDPC codes for spatial modulation systems over uncorrelated Rayleigh fading channels was proposed. The information sequence is first split into two subsequences, one for conventional signal modulation and the other for the spatial dimension of spatial modulation. One LDPC code is used independently in each subsequence. The code rates are chosen according to mutual information expressions of the corresponding component channels, derived in this work.

In the receiver, for the MAP detection processes, we proposed an iteration between the SPA decoders corresponding to the spatial and signal vectors. A gain in the overall system performance is obtained with this messages exchange between decoders.

Simulation results showed that the proposed two-layer total system presents better BER performance than the LDPC-SM one-layer system, the LDPC-SM two-layer partial system with iteration between decoders, and the LDPC-SM two-layer total system without iteration between decoders.

\section{REFERENCES}

[1] G. J. Foschini and M. J. Gans, "On Limits of Wireless Communications in a Fading Environment when Using Multiple Antennas," in Wirel. Pers. Commun. vol. 6, no. 3, pp. 311-335, 1998, doi: 10.1023/A:1008889222784.

[2] J. Mietzner, R. Schober, L. Lampe, W. H. Gerstacker and P. A. Hoeher, "Multiple-antenna techniques for wireless communications a comprehensive literature survey," in IEEE Communications Surveys and Tutorials, vol. 11, no. 2, pp. 87-105, Second Quarter 2009, doi: 10.1109/SURV.2009.090207.

[3] V. Tarokh, N. Seshadri and A. R. Calderbank, "Space-time codes for high data rate wireless communication: performance criterion and code construction," in IEEE Transactions on Information Theory, vol. 44, no. 2, pp. 744-765, Mar 1998, doi: 10.1109/18.661517. 
[4] S. M. Alamouti, "A simple transmit diversity technique for wireless communications," in IEEE Journal on Selected Areas in Communications, vol. 16, no. 8, pp. 1451-1458, Oct 1998, doi: 10.1109/49.730453.

[5] P. W. Wolniansky, G. J. Foschini, G. D. Golden and R. A. Valenzuela, "V-BLAST: an architecture for realizing very high data rates over the rich-scattering wireless channel," URSI International Symposium on Signals, Systems, and Electronics. Conference Proceedings (Cat. No.98EX167), Pisa, pp. 295-300, 1998, doi: 10.1109/ISSSE.1998.738086.

[6] R. Mesleh, H. Haas, C. W. Ahn and S. Yun, "Spatial Modulation - A New Low Complexity Spectral Efficiency Enhancing Technique," First International Conference on Communications and Networking in China, Beijing, pp. 1-5, 2006, doi: 10.1109/CHINACOM.2006.344658.

[7] M. Di Renzo, H. Haas, A. Ghrayeb, S. Sugiura and L. Hanzo, "Spatial Modulation for Generalized MIMO: Challenges, Opportunities, and Implementation," in Proceedings of the IEEE, vol. 102, no. 1, pp. 56103, Jan. 2014, doi: 10.1109/JPROC.2013.2287851.

[8] R. Mesleh, M. D. Renzo, H. Haas and P. M. Grant, "Trellis Coded Spatial Modulation," in IEEE Transactions on Wireless Communications, vol. 9, no. 7, pp. 2349-2361, July 2010, doi: 10.1109/TWC.2010.07.091526.

[9] E. Başar, U. Aygolu, E. Panayirci and H. V. Poor, "New Trellis Code Design for Spatial Modulation," in IEEE Transactions on Wireless Communications, vol. 10, no. 8, pp. 2670-2680, August 2011, doi: 10.1109/TWC.2011.061511.101745.

[10] C. Vladeanu, "Turbo trellis-coded spatial modulation," in IEEE overall Communications Conference (GLOBECOM), Anaheim, CA, pp. 40244029, 2012, doi: 10.1109/GLOCOM.2012.6503746.

[11] H. Liu, J. Zheng, J. Dou and B. Bai, "EXIT-chart-based LDPC code design for spatial modulation," in 4th IEEE International Conference on Information Science and Technology, Shenzhen, pp. 557-560, 2014, doi: 10.1109/ICIST.2014.6920539.

[12] X. Jiang, Y. Zheng, W. Chen, M. Wen and J. Li, "Two-Layer LDPC Codes for Low Complexity ML Detection in GSM Systems," in IEEE Wireless Communications Letters, vol. 7, no. 3, pp. 408-411, June 2018, doi: 10.1109/LWC.2017.2780080.

[13] D. Feng, H. Xu, J. Zheng and B. Bai, "Nonbinary LDPC-Coded Spatial Modulation," in IEEE Transactions on Wireless Communications, vol. 17, no. 4, pp. 2786-2799, April 2018, doi: 10.1109/TWC.2018.2803170.

[14] L. Wang, C. Liang, Z. Yang and X. Ma, "Two-Layer Coded Spatial Modulation With Block Markov Superposition Transmission," in IEEE Transactions on Communications, vol. 64, no. 2, pp. 643-653, Feb. 2016 , doi: 10.1109/TCOMM.2015.2506170.

[15] S. Zhao, X. Ma, Q. Huang and B. Bai, "Recursive Block Markov Superposition Transmission of Short Codes: Construction, Analysis, and Applications," in IEEE Transactions on Communications, vol. 66, no. 7, pp. 2784-2796, July 2018, doi: 10.1109/TCOMM.2018.2796607.

[16] R. G. Gallager, Low-Density Parity-Check Codes. Cambridge,MA, USAMIT Press, 1963.

[17] Sae-Young Chung, G. D. Forney, T. J. Richardson and R. Urbanke, "On the design of low-density parity-check codes within $0.0045 \mathrm{~dB}$ of the Shannon limit," in IEEE Communications Letters, vol. 5, no. 2, pp. 58 60, Feb 2001, doi: 10.1109/4234.905935.

[18] L. Wang and X. Ma, "Coded transceive spatial modulation with block Markov superposition transmission," in Electronics Letters, vol. 53, no. 10, pp. 657-659, 511 2017, doi: 10.1049/el.2016.4763.

[19] Z. Li et al., "Efficient encoding of quasi-cyclic low-density parity-check codes," in IEEE Transactions on Communications, v. 54, n. 1, p. 71-81, Jan 2006, doi: 10.1109/GLOCOM.2005.1577844.

[20] T. J. Richardson and R. L. Urbanke, "Efficient encoding of low-density parity-check codes," in IEEE Transactions on Information Theory, vol. 47, no. 2, pp. 638-656, Feb 2001, doi: 10.1109/18.910579.

[21] T. Zhang and K. K. Parhi, "A class of efficient-encoding generalized low-density parity-check codes," in IEEE International Conference on Acoustics, Speech, and Signal Processing. Proceedings (Cat. No.01CH37221), Salt Lake City, UT, USA, vol.4, pp. 2477-2480, 2001, doi: 10.1109/ICASSP.2001.940503.

[22] A. P. Legg, "Codificação LDPC para aplicações em códigos de barra 2D coloridos," PhD thesis, UFSC, Florianópolis, BR, 2011.

[23] C. Zhixiong and Y. Jinsha, "A Construction of Linearly Encodable QC-LDPC Codes by Grouping Cyclic Shift and Block Elimination," in ISECS International Colloquium on Computing, Communication, Control, and Management, Guangzhou, pp. 304-308, 2008, doi: 10.1109/CCCM.2008.174.

[24] Y. Yang and B. Jiao, "Information-guided channel-hopping for high data rate wireless communication," in IEEE Communications Letters, vol. 12, no. 4, pp. 225-227, April 2008, doi: 10.1109/LCOMM.2008.071986.
[25] X. Guan, Y. Cai and W. Yang, "On the Mutual Information and Precoding for Spatial Modulation with Finite Alphabet," in IEEE Wireless Communications Letters, vol. 2, no. 4, pp. 383-386, August 2013, doi: 10.1109/WCL.2013.050613.120923.

[26] Z. Yang, C. Liang, X. Xu, and X. Ma, "Block Markov superposition transmission with spatial modulation," in IEEE Communications Letters, vol. 3, no. 6, pp. 565-568, Dec. 2014, doi: 10.1109/LWC.2014.2349934.

[27] T. M. Cover and J. A. Thomas, Elements of Information Theory, 2nd ed, Hoboken, NJ, USA: Wiley, 2006.

[28] S. U. Hwang et al., "Soft-Output ML Detector for Spatial Modulation OFDM Systems," IEICE Electronics Express, vol. 6, no. 19, pp. 14261431, Oct. 2009, doi: 10.1587/elex.6.1426.

[29] C. Li, Y. Cheng, Y. Zhang and Y. Huang, "Low-complexity soft-output detectors for LDPC coded spatial modulation systems," 2015 International Conference on Wireless Communications and Signal Processing (WCSP), Nanjing, pp. 1-6, 2015, doi: 10.1109/WCSP.2015.7341061.

[30] A. Goldsmith, Wireless Communications, Cambridge University Press, New York, NY, USA, 2005.

[31] J. G. Proakis, M. Salehi, Digital Communications, 5th ed. New York, NY, USA:McGraw-Hill, 2008.

[32] I. E. Bocharova, B. D. Kudryashov, and R. Johannesson, "Searching for binary and nonbinary block and convolutional LDPC codes," in IEEE Trans. Inform. Theory, vol. 62, no. 1, pp. 163-183, Jan. 2016, doi: 10.1109/TIT.2015.2496213.

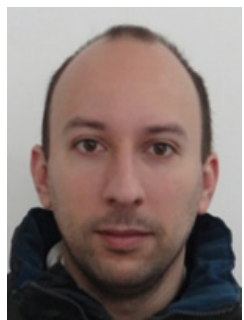

José Clair Menezes Júnior was born in Santa Maria - RS, Brazil, in 1987. He received the B.Sc. and M.Sc. degrees from Federal University of Santa Maria (UFSM), Santa Maria, RS, Brazil, in 2012 and 2014. He is currently a doctoral student of Electrical Engineering at UFSC and since 2016 he is Professor at Federal Institute Sul-rio-grandense (IFSul). He is member of Communications Research Group (GPqCom), Florianópolis, Brazil. His research interests lie in the areas of wireless communication, channel coding and signal processing.

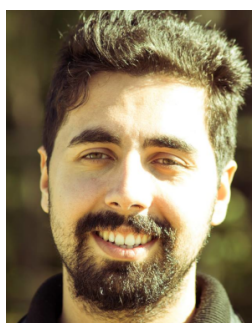

Bruno Fontana da Silva received the B.Sc. degree in Electrical Engineering from the Federal University of Santa Maria (UFSM), Santa Maria (RS), Brazil, in 2013, and his M.Sc. degree from the Federal University of Santa Catarina (UFSC), Florianópolis (SC), Brazil, in 2015, where he is currently pursuing the Ph.D. degree. Currently, he is also an assistant professor at the Instituto Federal Sul-rio-grandense (IFSul), Sapiranga (RS), Brazil. Mr. da Silva has been funded by National Council for Scientific and Technological Development (CNPq) with scholarships 132030/2013-6, 141161/2016-7 and 204676/20185 . His research interests include channel coding, MIMO communications, low-complexity receivers and multiple access. 


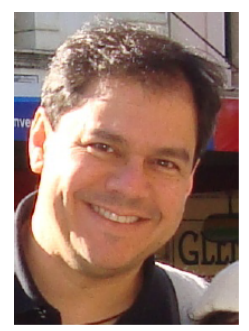

Bartolomeu F. Uchôa-Filho (S'94 - M'96 - SM'12) was born in Recife, Brazil, in 1965. He received the B.S.E.E. degree from the Federal University of Pernambuco (UFPE), Recife, Brazil, in 1989; the M.S.E.E. degree from the State University of Campinas (UNICAMP), Campinas, Brazil, in 1992; and the $\mathrm{Ph} . \mathrm{D}$. degree in electrical engineering from the University of Notre Dame, Notre Dame, Indiana, U.S.A., in 1996. During 1997-1999, he held a Postdoctoral positionat the State University of Campinas. From August 1999 to January 2000 he was a Visiting Researcher in the Department of Electrical Engineering, Federal University of Santa Catarina, Florianópolis, Brazil. Since February 2000 he has been with the same Department, where he is a Full Professor. From March 2009 to February 2010, he was a Post-Doctoral Fellow at the University of Sydney, Australia. From April to June 2017, he had a joint appointment as Senior Research Fellow in the Laboratoire des Signaux et Systèmes (L2S), Centrale-Supélec, and in the Laboratoire de Traitement du Signal et Architectures Électroniques (LAETITIA), Conservatoire National des Arts et Métiers (CNAM), both in France. His research interests are in the area of coding and information theory, with applications to digital communications systems. Dr. Uchôa-Filho is an IEEE Senior Member, a Senior Member of the Brazilian Telecommunications Society (SBrT), a member of Eta Kappa $\mathrm{Nu}$, the IEEE Information Theory Society, the IEEE Communications Society, and the ARC Communications Research Network (ACoRN, Australia). For the 2003-2004 period, he served as the Editor-in-Chief for the Journal of the Brazilian Telecommunications Society. During 2011-2014, he was an Associate Editor of Information and Coding Theory for the Journal of Communication and Information Systems (JCIS). Since 2016 he has been an Associate Editor of Physical Communication (Elsevier) and Digital Signal Processing (Elsevier). He was the Technical Program Committee Co-Chair of the 27th Brazilian Telecommunications Symposium (SBrT'09), the TrackChair of the Information Theory and Coding Track of the 2014 IEEE/SBrT International Telecommunications Symposium (ITS'14), and has also served as TPC member of several national and international symposia. He is a Research Productivity Fellow (Level 1C) of the CNPq (the Brazilian National Council for Scientific and Technological Development, Ministry of Science and Technology). 